\title{
Standardizing Zebrafish Behavioral Paradigms Across Life Stages: An Effort Towards Translational Pharmacology
}

\author{
Barbara Dutra Petersen ${ }^{1,2}$, Kanandra Taisa Bertoncello ${ }^{2,3}$ and Carla Denise Bonan ${ }^{1,2,3 *}$ \\ ${ }^{1}$ Programa de Pós-Graduação em Medicina e Ciências da Saúde, Escola de Medicina, Pontifícia Universidade Católica do Rio \\ Grande do Sul, Porto Alegre, Brazil, ${ }^{2}$ Laboratório de Neuroquímica e Psicofarmacologia, Escola de Ciências da Saúde e da Vida, \\ Pontifícia Universidade Católica do Rio Grande do Sul, Porto Alegre, Brazil, ${ }^{3}$ Programa de Pós-Graduação em Biologia Celular e \\ Molecular, Escola de Ciências da Saúde e da Vida, Pontifícia Universidade Católica do Rio Grande do Sul, Porto Alegre, Brazil
}

OPEN ACCESS

Edited by: Yuhei Nishimura, Mie University, Japan

Reviewed by: Rebecca Ann Wingert, University of Notre Dame,

United States

*Correspondence:

Carla Denise Bonan cbonan@pucrs.br

Specialty section:

This article was submitted to Translational Pharmacology,

a section of the journal

Frontiers in Pharmacology

Received: 13 December 2021 Accepted: 03 January 2022 Published: 20 January 2022

Citation:

Petersen $B D$, Bertoncello $K T$ and Bonan CD (2022) Standardizing Zebrafish Behavioral Paradigms Across Life Stages: An Effort Towards

Translational Pharmacology. Front. Pharmacol. 13:833227. doi: 10.3389/fphar.2022.833227
Zebrafish is a prominent vertebrate model, with many of its advantages related to its development, life cycle, and translational ability. While a great number of behavioral phenotypes and tasks to evaluate them are available, longitudinal studies across zebrafish life stages are scarce and made challenging because of the differences between protocols and endpoints assessed at each life stage. In this mini review, we highlight the relevance that longitudinal studies could have for neurobehavioral pharmacology using this model. We also present possible strategies to standardize behavior endpoints in domains related to human diseases throughout the life cycle, especially between larvae and adult fish. Furthermore, we discuss the remaining difficulties of these analyses and explore future advances needed to bridge this knowledge gap.

Keywords: zebrafish, behavioral tests, ontology, anxiety, exploratory behavior, sociability, cognition (Min. 5-Max. 8)

\section{INTRODUCTION}

Zebrafish is a widely used vertebrate model species in research fields such as genetics, pharmacology, developmental biology, neuroscience, among others (Senger et al., 2004; Egan et al., 2009; Spence et al., 2011; Siebel et al., 2015). As an alternative to rodents, zebrafish has numerous advantages. Their habits are diurnal, they reproduce throughout the year and produce a large number of eggs with each reproduction (Kalueff et al., 2014). Furthermore, external development and the absence of parental care reduce the epigenetic influences of parental animals (Mushtaq et al., 2013) and allow manipulations during embryonic development (Kalueff et al., 2014). Initial development is rapid - embryogenesis lasts approximately $24 \mathrm{~h}$ (Dahm et al., 2006) - with most neurochemical pathways already functional, although immature, at around $60 \mathrm{~h}$ post-fertilization (hpf) (Arenzana et al., 2005; Sallinen et al., 2009; Cocco et al., 2017; Monesson-Olson et al., 2018).

The high capacity of behavioral analysis is also a feature of this model. The importance of behavioral traits in research stems mostly from it being a functional readout of neural activity (Gerlai et al., 2000; Valente et al., 2012; Orger and De Polavieja, 2017). In this sense, although zebrafish and mammals have marked anatomical differences in the structural organization of the central nervous system (CNS) (Wullimann and Mueller, 2004; Sager et al., 2010), there is a strong functional homology, reflected in the similarity of effects in response to exposure to compounds between zebrafish and mammals (Bailey et al., 2013) and in the successful adaptation of many behavioral 
paradigms of rodents to this species (Kalueff et al,, 2014). In zebrafish, behavioral assessments can be performed as early as $17 \mathrm{hpf}$, still inside the eggs (Kokel et al., 2010), and more complex tasks are available when the animals reach a state of freeswimming and active search for food within 5 days after fertilization (dpf) (Neuhauss, 2003). As fish develops, behaviors increase in complexity. Most studies focus on either larval or adult stages, with around 190 distinct behavioral phenotypes already described for zebrafish (Kalueff et al., 2013). These phenotypes combined can be arranged in behavioral domains (Kalueff et al., 2013; Kalueff et al., 2014) and serve as significant endpoints in biomedical and human disease translational research (Ninkovic and Bally-Cuif, 2006; Kalueff et al., 2014; Fontana et al., 2018), toxicology, and pharmacology (Goldsmith, 2004; Bailey et al., 2013; MacRae and Peterson, 2015; Costa et al., 2020).

Granting all this, translationality of behavior across life stages in zebrafish is still poorly explored, mainly because paradigms for larvae and adult fish differ in form and endpoints, thus not allowing direct comparisons between stages. In this review, we will discuss the significance of this gap focusing on behavioral responses to pharmacological interventions, the currently used paradigms to each life stage and behavioral domain, the alternative paradigms that can potentially be used across stages to close this gap, and the drawbacks and benefits of each strategy.

\section{VARIABILITY OF PHARMACOLOGICAL RESPONSES BY LIFE STAGES}

Age is a factor that might influence zebrafish behavior and its pharmacological responses, especially in early life stages when results reflect specific time windows in which the neural systems are not yet mature (Orger and De Polavieja, 2017). Despite the difficulties for transposing results across ages, posed by the variations of endpoints, studies of chemicals in varying developmental stages indicate the advantages zebrafish could have if this gap was diminished. One drug that was extensively studied throughout zebrafish development is the NMDA receptor antagonist MK-801. In general, neurobehavioral actions of this drug are conserved in zebrafish (Chen et al., 2010; Sison and Gerlai, 2011), and the findings are comparable to those previously described in rodents (Löscher and Hönack, 1992). Some studies using MK-801 demonstrate different behavioral responses of zebrafish during its development.

Age-related locomotor responses to $\mathrm{MK}-801$ were reported in zebrafish (Menezes et al., 2015). In this study, the locomotor response of zebrafish to MK-801 through the development, from $30 \mathrm{dpf}$ to 2 years post-fertilization (ypf) was evaluated. Distance traveled showed that 30 -day-old zebrafish did not respond to MK-801, whereas in animals aged $60 \mathrm{dpf}, 120 \mathrm{dpf}$, and $2 \mathrm{ypf}$, the antagonist promoted an increase in locomotor activity at the concentration of $5 \mu \mathrm{M}$. When the NMDA receptor subunit gene expression was analyzed through the development (7 dpf-2 ypf), the results showed variations in the expression of NR1.1 and NR2A.2 and NR2C.1 subunits throughout the development.
With this, the changes in locomotor responses to MK-801 exposure through the development could be a consequence of differential NMDA receptor subunit expression (Menezes et al., 2015). This result of developmental responses to MK-801 highlights the significance of cross-ages studies, and how this model can uncover characteristics of neural development useful for pharmacological interventions.

The effects of MK-801 were assessed in more complex behaviors. Social interactions variations in response to MK801 demonstrated that zebrafish submitted to a preference/ interaction assay show a social behavior that starts to emerge by 2 weeks post-fertilization (wpf) and is robust at $3 \mathrm{wpf}$ (Dreosti et al., 2015). At this point ( $3 \mathrm{wpf}$ ), acute treatment with $100 \mu \mathrm{M}$ MK-801 disrupted social preference, as was previously shown in adults (Sison and Gerlai, 2011). This suggests that blocking NMDA receptors interferes with the circuitry required for social interactions, both in larva and in adult fish. Cognition, as tested with novel object recognition paradigms, was also evaluated both in larvae and adult zebrafish, demonstrating different responses to MK-801. While in larvae MK-801 did not affect memory retrieval in short- or long-term memory assay in zebrafish at $10 \mathrm{dpf}$ (Andersson et al., 2015), MK-801 impaired object location memory in adult zebrafish on a novel object recognition test (Gaspary et al., 2018). It indicates dependent differences in the action of the antagonist according to age, once in zebrafish larvae MK-801 did not influence the retrieval of memories obtained before the treatment, as was expected by previous adult outcomes.

The studies described here serve as an example and highlight the importance of a better understanding of behavioral phenotypes at different stages of zebrafish development. Results to MK-801 vary depending on age, the complexity of behavior, and endpoint assessed. Therefore, assessing equivalent endpoints throughout life can potentially bring zebrafish pharmacology to a new level. It may facilitate the observations of how responses develop and increase zebrafish potential as a translational model that enables the study of ontogenic changes of molecular drug targets.

\section{ZEBRAFISH BEHAVIORAL PARADIGMS}

\subsection{General Behavior and Anxiety}

General behavior and anxiety in zebrafish are generally evaluated together, although specific tests to evaluate anxiety are available. Regarding the general well-being of zebrafish, this assessment can be performed during behavioral tasks, with the main parameters common to the various tasks being total distance traveled, erratic movements (usually measured by the angle of rotation), and immobility (Westerfield, 2000; Kalueff and Stewart, 2012; Kalueff et al., 2013). The animal's body position can be observed during these tests and indicate disease or exposure responses to compounds (Kalueff et al., 2013). The assessment of these parameters together can lead to the characterization of complex behaviors such as hypolocomotion, hyperlocomotion, sickness behavior, motor incoordination, among others (Kalueff et al., 2013). 
Although these behavioral phenotypes can be evaluated in a wide variety of tests, the general aspects of zebrafish locomotion are usually analyzed in the novel tank or open field tests (Bridi et al., 2017; Nabinger et al., 2020; Petersen et al., 2021). In adults, novel tank and open tank tests are performed in a rectangular or trapezoid aquarium and the typical zebrafish behavior is to stay at the bottom of the apparatus for the first few minutes of testing, followed by top exploration, a phenomenon called bottomdwelling (Kalueff et al., 2013). Animals also tend to exhibit thigmotaxis, that is, to remain close to the walls of the apparatus, although this is rarely assessed by the difficulty of measuring time at the bottom/top and thigmotaxis simultaneously with a single camera (Kalueff and Stewart, 2012). In larvae, the paradigm used to assess exploration is usually carried out with the camera below or above 24 or 96well plates, so that thigmotaxis, but not depth preference, is assessed (Creton, 2009). In both larvae and adults, locomotion in these scenarios follows a sinusoidal oscillatory pattern, with alternating phases of higher and lower velocities with a frequency of approximately $5 \mathrm{~min}$ (Kalueff and Stewart, 2012; Kalueff et al., 2013).

With this, the main differences in the above-mentioned paradigms for larvae and adults are the positioning of the camera, which impact the endpoints that can be retrieved later with automated analysis software (bottom-dwelling or thigmotaxis), and the form of the apparatus (rectangular or round). Although unusual, the rectangular novel tank test has recently been adapted for older larvae (Golla et al., 2020). In this variation, fish were filmed in groups, and bottom dwelling was measured not by time but by ratio. Despite this, the protocol can be used as an alternative to access larvae in a similar way to adult fish. The main con of this proposal would be to lose the assessment of thigmotaxis.

Another alternative regarding standardization of general behavior across life stages could be filming adult fish from above, in round tanks. This alternative is especially promising for pharmacological studies, once it allows coupling with the Light Motor Response (LMR) test and reduces the number of animals used. The LMR test, also called motor response to light/ dark transitions, is widely used in zebrafish larvae, mainly due to its high sensitivity to neuroactive compounds (Emran et al., 2008; Legradi et al., 2014; Klüver et al., 2015; Leuthold et al., 2019), and has already been adapted for adult animals (Shao et al., 2017). For the LMR, after habituation to the tank or well, light condition is changed periodically from light to darkness and behavior is recorded with an infrared enabled camera, with different number and length of cycles described in the literature (Emran et al., 2008; Legradi et al., 2014; Klüver et al., 2015; Leuthold et al., 2019). Alike the novel/open tank, the parameters retrieved are distance traveled, erratic movements, thigmotaxis, immobility, and more, only the analysis is coupled by light condition, hence there are typical behavioral changes exhibited by animals in dark and light environments, and in the transitions between these two conditions.

The three paradigms discussed (rectangular novel tank, round novel tank, and LMR) can also be used to evaluate anxiety-like states in zebrafish. Stress and anxiety models are widely used in research related to neuroscience and as a behavioral parameter for drug discovery studies with effects on the CNS (Kysil et al., 2017). In other experimental models, but especially in zebrafish, the behavioral phenotypes of fear, stress, and anxiety are confusing, and species-specific patterns that help in their differentiation are not well established (Maximino et al., 2010a). Thus, we will use the term "anxiety" to describe the set of these phenotypes. In the novel tank test, anxiety is assessed together with exploratory behavior, by observing the parameters: the latency to swim to the upper half of the tank, the number of transitions between the lower and upper half, the time in each of these zones, the instances of erratic movements and immobility and freezing responses (Maximino et al., 2010a; Kysil et al., 2017). Finally, regarding outcomes that can be evaluated in non-specific paradigms for anxiety, thigmotaxis (time, frequency, crossings between center and borders of the well) is used as an parameter for indicating anxiety in larvae, and in some tests used to assess cognition based on aversive stimuli and also on light transition tests (Maximino et al., 2010a; Schnörr et al., 2012).

Among the specific tests for anxiety, the most recognized is the light/dark preference test, based on the animals' natural scototaxis. This test has already been adapted for different stages of development and presented with some methodological variations (Serra et al., 1999; Maximino et al., 2010b; Stewart et al., 2011; Golla et al., 2020). In general, the paradigm consists of placing the animal individually in an apparatus with a zone with white walls and floor and another zone with black walls and floor. The parameters used to measure anxiety are number of transitions between zones, latency for the aversive zone, number of risk assessment episodes (rapid entries, without permanence in the aversive zone), thigmotaxis in the aversive zone, time in the aversive zone, number of freezing episodes and erratic movements (Maximino et al., 2010b; Steenbergen et al., 2011; Kysil et al., 2017). The main variation in responses to being placed in this half-black, half-white tank between life stages is that while animals in the larval stage prefer light zones, juvenile and adult animals prefer dark zones (Kalueff et al., 2013; Kysil et al., 2017). Exactly when this transition of preference occurs is still an unknown point. Thus, to enable comparisons between life stages, the researcher must be aware of the preference the animals exhibit and evaluate the outcomes about the zone that the animal tends to avoid (light for adults, dark for larvae) or prefer. Another point of care is the variation of the paradigm that will be used, depending on the age and size of animals. Given that the size of the tank should be adapted to the animal's size (Buske and Gerlai, 2011) when using smaller animals such as young larvae, the use of sliding doors may be non-practical, thus the paradigm of Serra et al. (1999) should be preferred.

\subsection{Cognition}

The cognition domain comprises the processes involved in learning and memory. The diversity of behavioral paradigms aimed to study this domain in zebrafish is high, however, the most used tasks are based on conditioning to aversive or rewarding stimuli, or based on the natural preference of these animals for new objects or situations (Blank et al., 2009; Cognato et al., 2012; 
Gaspary et al., 2018; Yashina et al., 2019). Concerning to the tasks based on classical or operant conditioning, the main stimuli are usually aversive, most notably the use of electric shocks (Blank et al., 2009; Hinz et al., 2013; Harmon et al., 2017; Yashina et al., 2019). This type of test is usually performed in multiple sessions and generates long-term memories, and the main outcomes measured are permanence in the zone considered safe or the latency to enter the zone where the animal previously received the aversive stimulus (Blank et al., 2009; Valente et al., 2012; Yashina et al., 2019).

How early zebrafish can learn and retrieve memories is still a point of debate. Classical conditioning tasks have been successfully performed in animals in the early stages of development at $6 \mathrm{dpf}$ (Aizenberg and Schuman, 2011; Harmon et al., 2017) and $3 \mathrm{wpf}$ (Valente et al., 2012; Matsuda et al., 2017). Regarding operant conditioning, Yashina et al. (2019) tested animals at 7,14 , and $21 \mathrm{dpf}$, demonstrating that learning varies according to animal size at 7 and $14 \mathrm{dpf}$, suggesting that body length, not age in days, reflects the brain development necessary for learning in this age group. The duration of memories acquired through conditioning protocols also seems to depend on the test used and the age of the animals (Roberts et al., 2013; Yashina et al., 2019). For short-term memories, the Y-maze is used in adult animals (Cognato et al., 2012; Fontana et al., 2021; Petersen et al., 2021). This paradigm is based on visual cues and the preference of zebrafish for novel situations to form a spatial memory of animals (Cognato et al., 2012). The Y-Maze has not yet been characterized in animals in their early stages of development; however, a paradigm based on visual cues and the presence of a new object in $10 \mathrm{dpf}$ animals was characterized, demonstrating that older larvae can learn spatial tasks (Andersson et al., 2015).

In such a case, studying cognitive alterations across zebrafish development is still a challenge. Although classical conditioning appears to be the safest option, results in young larvae are inconsistent depending on the size of animals, inter-trial interval, and specific paradigm (Valente et al., 2012; Harmon et al., 2017; Yashina et al., 2019). Given the importance of cognitive symptoms in many health conditions, the adaptation and validation of a paradigm suited for such purposes present as major gap in the behavioral neuroscience of zebrafish.

\subsection{Sociability}

The social behavior of zebrafish encompasses the interaction with conspecifics, aggression, and sexual behaviors (Kalueff et al., 2013), all of which are highly conserved in this species (Scott and Sloman, 2004; Stednitz and Washbourne, 2020). So far, studies related to this domain have focused on non-sexual interaction and aggressiveness (Buske and Gerlai, 2011). This behavioral domain starts to develop a little further in development. Non-sexual social interaction encompasses a set of behaviors that develop from the preference for conspecifics at around $3 \mathrm{wpf}$, and around $45 \mathrm{dpf}$ culminates in ordered swimming in groups (known as shoaling) (Engeszer et al., 2007; Buske and Gerlai, 2011; Dreosti et al., 2015; Stednitz and Washbourne, 2020). The main test for the evaluation of this characteristic is the social interaction test, where the animals are exposed to the vision of conspecifics and their proximity and distance from the zone close to them is evaluated. Factors that influence the results and may be crucial in the observed age differences are the number of fish tested and stimulus fish used, the pairing or not of the size between test fish and stimulus fish, and the living conditions of the animals before the test (Dreosti et al., 2015; Groneberg et al., 2020; Stednitz and Washbourne, 2020). With regard to aggressive behavior, using the tilted mirror and dyadic struggles paradigms, respectively, Carreño Gutiérrez et al. (2018) and Ricci et al. (2013) found results suggesting that the onset of aggressive behavior occurs around $28 \mathrm{dpf}$.

The current data suggest that social behaviors in zebrafish can be evaluated only at early juvenile stages. It is unknown, however, if more subtle indications of sociability exist and can be explored earlier on. Nonetheless, with the current tests available, the social interaction/preference and the tilted mirror aggression paradigms depend less on group behavior than the shoaling and dyadic fight variations, as well as social preference responses develop earlier than the shoaling responses.

\section{CONCLUSION AND FUTURE PERSPECTIVES}

Since the 1970s, when zebrafish started to be used as an animal model, advances have been made in decoupling interfering variables in studies. Some examples of this are the relatively recent availability of isogenic strains (Lange et al., 2013; Kalueff et al., 2014), and the funding of researcher networks that aim to reduce environmental variability between laboratories, such as ZFIN and ZIRC. These and other initiatives have brought zebrafish to a new light as a translational model. However, unlike other animal models, longitudinal studies focused on behavior that could help to uncover developmental pharmacological mechanisms, developmental origins of health and disease, effects of epigenetics and other environmental processes, normal aging, as well as transgenerational effects are still scarce (Lange et al., 2013; Kalueff et al., 2014; Tal et al., 2020). In this review, we aimed to highlight the significance of such studies in light of pharmacological interventions and to examine possible adaptations in already established behavioral paradigms that would allow such longitudinal studies to be feasible throughout zebrafish life cycle. However, questions around this subject are left open.

First, while some of the studies presented here point to the feasibility of adapting tasks across ages, the face and construct validity of these strategies must be tested. Even more so given that other developmental stages such as the juvenile and old-age stages are less characterized than larvae and adults. Secondly, for some behavioral domains, such as cognition and sociability presented here, the adaptation of existing protocols is a challenging task. This is due mainly because of inconsistencies of results at early life stages, about when these behaviors start. To bridge this gap, a close observation of some points must be made. Early neural development in zebrafish is highly dependent on environmental factors such as temperature and housing density (Parichy et al., 2009). Data suggest that body length rather than age in days post-fertilization is a better indicator of the neural stage (Polverino et al., 2016; Yashina et al., 2019; Stednitz and 
Washbourne, 2020). Therefore, standardizing behavioral paradigms in zebrafish can also serve as a tool to find sensitive time points to assess different behavioral domains in this species. It is also important to note that while behavioral phenotypes are a complex product of CNS activity (Stewart et al., 2014), the effort to standardize behavioral paradigms must be coupled with the studies on the pharmacokinetics and pharmacodynamics of drugs and how these mechanisms develop along with the developing brain. Finally, it would be beneficial for the field if for the proposal and construction of new paradigms that the viability of its use across the developmental stages of zebrafish may be considered.

\section{AUTHOR CONTRIBUTIONS}

$\mathrm{BP}$ and $\mathrm{KB}$ conceptualized the manuscript and retrieved the literature, $\mathrm{BP}, \mathrm{KB}$, and $\mathrm{CB}$ drafted the manuscript and revised

\section{REFERENCES}

Aizenberg, M., and Schuman, E. M. (2011). Cerebellar-Dependent Learning in Larval Zebrafish. J. Neurosci. 31 (24), 8708-8712. doi:10.1523/ JNEUROSCI.6565-10.2011

Andersson, M. A., Ek, F., and Olsson, R. (2015). Using Visual Lateralization to Model Learning and Memory in Zebrafish Larvae. Sci. Rep. 5, 8667-7. doi:10.1038/srep08667

Arenzana, F. J., Clemente, D., Sánchez-González, R., Porteros, A., Aijón, J., and Arévalo, R. (2005). Development of the Cholinergic System in the Brain and Retina of the Zebrafish. Brain Res. Bull. 66 (4), 421-425. doi:10.1016/ j.brainresbull.2005.03.006

Bailey, J., Oliveri, A., and Levin, E. D. (2013). Zebrafish Model Systems for Developmental Neurobehavioral Toxicology. Birth Defects Res. C Embryo Today 99 (1), 14-23. doi:10.1002/bdrc. 21027

Blank, M., Guerim, L. D., Cordeiro, R. F., Vianna, M. R., and Vianna, M. R. M. (2009). A One-Trial Inhibitory Avoidance Task to Zebrafish: Rapid Acquisition of an NMDA-Dependent Long-Term Memory. Neurobiol. Learn. Mem. 92 (4), 529-534. doi:10.1016/j.nlm.2009.07.001

Bridi, D., Altenhofen, S., Gonzalez, J. B., Reolon, G. K., and Bonan, C. D. (2017). Glyphosate and Roundup ${ }^{\circledR}$ Alter Morphology and Behavior in Zebrafish. Toxicology 392 (July), 32-39. doi:10.1016/j.tox.2017.10.007

Buske, C., and Gerlai, R. (2011). Shoaling Develops with Age in Zebrafish (Danio rerio). Prog. Neuropsychopharmacol. Biol. Psychiatry 35 (6), 1409-1415. doi:10.1016/j.pnpbp.2010.09.003

Carreño Gutiérrez, H., Vacca, I., Pons, A. I., and Norton, W. H. J. (2018). Automatic Quantification of Juvenile Zebrafish Aggression. J. Neurosci. Methods 296, 23-31. doi:10.1016/j.jneumeth.2017.12.012

Chen, J., Patel, R., Friedman, T. C., and Jones, K. S. (2010). The Behavioral and Pharmacological Actions of NMDA Receptor Antagonism are Conserved in Zebrafish Larvae. Int. J. Comp. Psychol. 23 (1), 82-90.

Cocco, A., Rönnberg, A. M., Jin, Z., André, G. I., Vossen, L. E., Bhandage, A. K., et al. (2017). Characterization of the $\gamma$-Aminobutyric Acid Signaling System in the Zebrafish (Danio $R$ Hamilton) Central Nervous System by Reverse Transcription-Quantitative Polymerase Chain Reaction. Neuroscience 343, 300-321. doi:10.1016/j.neuroscience.2016.07.018

Cognato, G. D. P., Bortolotto, J. W., Blazina, A. R., Christoff, R. R., Lara, D. R., Vianna, M. R., et al. (2012). Y-Maze Memory Task in Zebrafish (Danio R): The Role of Glutamatergic and Cholinergic Systems on the Acquisition and Consolidation Periods. Neurobiol. Learn. Mem. 98 (4), 321-328. doi:10.1016/j.nlm.2012.09.008

Costa, B. P. D., Moura, L. A., Pinto, S. A. G., Lima-Maximino, M., and Maximino, C. (2020). Zebrafish Models in Neural and Behavioral Toxicology across the Life Stages. Fishes 5 (3), 1-14. doi:10.3390/ fishes5030023 the manuscript. All authors edited, revised, and approved the final version of this review.

\section{FUNDING}

This study was funded in part by the Coordenação de Aperfeiçoamento de Pessoal de Nível Superior - Brasil (CAPES) - finance code 001, Conselho Nacional de Desenvolvimento Científico e Tecnológico (CNPq) (Proc. 420695/2018-4), Fundação de Amparo à Pesquisa do Estado do Rio Grande do Sul, and Instituto Nacional de Ciências e Tecnologia para Doenças Cerebrais, Excitotoxicidade e Neuroproteção. BP and CB are recipients of CNPq fellowship (Proc. 140312/2021-8) and research productivity grants (Proc. $304450 / 2019-7)$.

Creton, R. (2009). Automated Analysis of Behavior in Zebrafish Larvae. Behav. Brain Res. 203 (1), 127-136. doi:10.1016/j.bbr.2009.04.030

Dahm, R., Geisler, R., and Nüsslein-Volhard, C. (2006). "Zebrafish (Danio R) Genome and Genetics," in Reviews in Cell Biology and Molecular Medicine (Weinheim, Germany: Wiley-VCH Verlag GmbH \& Co. KGaA). doi:10.1002/ 3527600906.mcb.200400059

Dreosti, E., Lopes, G., Kampff, A. R., and Wilson, S. W. (2015). Development of Social Behavior in Young Zebrafish. Front. Neural Circuits 9, 39-9. doi:10.3389/ fncir.2015.00039

Egan, R. J., Bergner, C. L., Hart, P. C., Cachat, J. M., Canavello, P. R., Elegante, M. F., et al. (2009). Understanding Behavioral and Physiological Phenotypes of Stress and Anxiety in Zebrafish. Behav. Brain Res. 205 (1), 38-44. doi:10.1016/ j.bbr.2009.06.022

Emran, F., Rihel, J., and Dowling, J. E. (2008). A Behavioral Assay to Measure Responsiveness of Zebrafish to Changes in Light Intensities. J. Vis. Exp. 20, 1-6. doi:10.3791/923

Engeszer, R. E., Barbiano, L. A., Ryan, M. J., and Parichy, D. M. (2007). Timing and Plasticity of Shoaling Behaviour in the Zebrafish, Danio Rerio. Anim. Behav. 74 (5), 1269-1275. doi:10.1016/j.anbehav.2007.01.032

Fontana, B. D., Gibbon, A. J., Cleal, M., Sudwarts, A., Pritchett, D., Miletto Petrazzini, M. E., et al. (2021). Moderate Early Life Stress Improves Adult Zebrafish (Danio Rerio) Working Memory but Does Not Affect Social and Anxiety-Like Responses. Dev. Psychobiol 63 (1), 54-64. doi:10.1002/dev.21986

Fontana, B. D., Mezzomo, N. J., Kalueff, A. V., and Rosemberg, D. B. (2018). The Developing Utility of Zebrafish Models of Neurological and Neuropsychiatric Disorders: A Critical Review. Exp. Neurol. 299, 157-171. doi:10.1016/ j.expneurol.2017.10.004

Gaspary, K. V., Reolon, G. K., Gusso, D., and Bonan, C. D. (2018). Novel Object Recognition and Object Location Tasks in Zebrafish: Influence of Habituation and NMDA Receptor Antagonism. Neurobiol. Learn. Mem. 155, 249-260. doi:10.1016/j.nlm.2018.08.005

Gerlai, R., Lahav, M., Guo, S., and Rosenthal, A. (2000). Drinks Like a Fish: Zebra Fish (Danio Rerio) as a Behavior Genetic Model to Study Alcohol Effects. Pharmacol. Biochem. Behav. 67, 773-782. doi:10.1016/s0091-3057(00)00422-6

Goldsmith, P. (2004). Zebrafish as a Pharmacological Tool: The How, Why and When. Curr. Opin. Pharmacol. 4 (5), 504-512. doi:10.1016/j.coph.2004.04.005

Golla, A., Østby, H., and Kermen, F. (2020). Chronic Unpredictable Stress Induces Anxiety-Like Behaviors in Young Zebrafish. Sci. Rep. 10 (1), 10339-10410. doi:10.1038/s41598-020-67182-4

Groneberg, A. H., Marques, J. C., Martins, A. L., Diez del Corral, R., de Polavieja, G. G., and Orger, M. B. (2020). Early-Life Social Experience Shapes Social Avoidance Reactions in Larval Zebrafish. Curr. Biol. 30 (20), 4009-4021. doi:10.1016/j.cub.2020.07.088

Harmon, T. C., Magaram, U., McLean, D. L., and Raman, I. M. (2017). Distinct Responses of Purkinje Neurons and Roles of Simple Spikes during Associative Motor Learning in Larval Zebrafish. Elife 6, 1-26. doi:10.7554/eLife.22537 
Hinz, F. I., Aizenberg, M., Tushev, G., and Schuman, E. M. (2013). Protein Synthesis-Dependent Associative Long-Term Memory in Larval Zebrafish. J. Neurosci. 33 (39), 15382-15387. doi:10.1523/JNEUROSCI.0560-13.2013

Kalueff, A. V., and Stewart, A. M. (2012). Zebrafish Protocols for Neurobehavioral Research. Editors A. V. Kalueff and A. M. Stewart. 1st ed. (Totowa, NJ: Humana Press), 357.

Kalueff, A. V., Stewart, A. M., and Gerlai, R. (2014). Zebrafish as an Emerging Model for Studying Complex Brain Disorders. Trends Pharmacol. Sci. 35 (2), 63-75. doi:10.1016/j.tips.2013.12.002

Kalueff, A. V., Gebhardt, M., Stewart, A. M., Cachat, J. M., Brimmer, M., Chawla, J. S., et al. (2013). Towards a Comprehensive Catalog of Zebrafish Behavior 1.0 and Beyond. Zebrafish 10 (1), 70-86. doi:10.1089/ zeb.2012.0861

Klüver, N., König, M., Ortmann, J., Massei, R., Paschke, A., Kühne, R., et al. (2015). Fish Embryo Toxicity Test: Identification of Compounds with Weak Toxicity and Analysis of Behavioral Effects to Improve Prediction of Acute Toxicity for Neurotoxic Compounds. Environ. Sci. Technol. 49 (11), 7002-7011. doi:10.1021/acs.est.5b01910

Kokel, D., Bryan, J., Laggner, C., White, R., Cheung, C. Y., Mateus, R., et al. (2010). Rapid Behavior-Based Identification of Neuroactive Small Molecules in the Zebrafish. Nat. Chem. Biol. 6 (3), 231-237. doi:10.1038/nchembio.307

Kysil, E. V., Meshalkina, D. A., Frick, E. E., Echevarria, D. J., Rosemberg, D. B., Maximino, C., et al. (2017). Comparative Analyses of Zebrafish Anxiety-Like Behavior Using Conflict-Based Novelty Tests. Zebrafish 14 (3), 197-208. doi:10.1089/zeb.2016.1415

Lange, M., Neuzeret, F., Fabreges, B., Froc, C., Bedu, S., Bally-Cuif, L., et al. (2013). Inter-Individual and Inter-Strain Variations in Zebrafish Locomotor Ontogeny. PLoS One 8 (8), e70172. doi:10.1371/journal.pone.0070172

Legradi, J., el Abdellaoui, N., van Pomeren, M., and Legler, J. (2014). Comparability of Behavioural Assays Using Zebrafish Larvae to Assess Neurotoxicity. Environ. Sci. Pollut. Res. Int. 22 (21), 16277-16289. doi:10.1007/s11356-014-3805-8

Leuthold, D., Klüver, N., Altenburger, R., and Busch, W. (2019). Can Environmentally Relevant Neuroactive Chemicals Specifically Be Detected with the Locomotor Response Test in Zebrafish Embryos? Environ. Sci. Technol. 53 (1), 482-493. doi:10.1021/acs.est.8b04327

Löscher, W., and Hönack, D. (1992). The Behavioural Effects of MK-801 in Rats: Involvement of Dopaminergic, Serotonergic and Noradrenergic Systems. Eur. J. Pharmacol. 215 (2), 199-208.

MacRae, C. A., and Peterson, R. T. (2015). Zebrafish as Tools for Drug Discovery. Nat. Rev. Drug Discov. 14 (10), 721-731. doi:10.1038/nrd4627

Matsuda, K., Yoshida, M., Kawakami, K., Hibi, M., and Shimizu, T. (2017). Granule Cells Control Recovery from Classical Conditioned Fear Responses in the Zebrafish Cerebellum. Sci. Rep. 7 (1), 11865-11913. doi:10.1038/s41598-01710794-0

Maximino, C., de Brito, T. M., da Silva Batista, A. W., Herculano, A. M., Morato, S., and Gouveia, A. (2010). Measuring Anxiety in Zebrafish: A Critical Review. Behav. Brain Res. 214 (2), 157-171. doi:10.1016/j.bbr.2010.05.031

Maximino, C., Marques De Brito, T., Dias, C. A., Gouveia, A., and Morato, S. (2010). Scototaxis as Anxiety-Like Behavior in Fish. Nat. Protoc. 5 (2), 209-216. doi:10.1038/nprot.2009.225

Menezes, F. P., Kist, L. W., Bogo, M. R., Bonan, C. D., and Da Silva, R. S. (2015). Evaluation of Age-Dependent Response to NMDA Receptor Antagonism in Zebrafish. Zebrafish 12 (2), 137-143. doi:10.1089/zeb.2014.1018

Monesson-Olson, B., McClain, J. J., Case, A. E., Dorman, H. E., Turkewitz, D. R., Steiner, A. B., et al. (2018). Expression of the Eight GABAA Receptor a Subunits in the Developing Zebrafish Central Nervous System. PLoS One 13 (4), e0196083. doi:10.1371/journal.pone.0196083

Mushtaq, M. Y., Verpoorte, R., and Kim, H. K. (2013). Zebrafish as a Model for Systems Biology. Biotechnol. Genet. Eng. Rev. 29 (2), 187-205. doi:10.1080/ 02648725.2013.801238

Nabinger, D. D., Altenhofen, S., Peixoto, J. V., da Silva, J. M. K., Gerlai, R., and Bonan, C. D. (2020). Feeding Status Alters Exploratory and Anxiety-like Behaviors in Zebrafish Larvae Exposed to Quinpirole. Prog. Neuropsychopharmacol. Biol. Psychiatry 108, 110179. doi:10.1016/ j.pnpbp.2020.110179

Neuhauss, S. C. (2003). Behavioral Genetic Approaches to Visual System Development and Function in Zebrafish. J. Neurobiol. 54 (1), 148-160. doi:10.1002/neu. 10165
Ninkovic, J., and Bally-Cuif, L. (2006). The Zebrafish as a Model System for Assessing the Reinforcing Properties of Drugs of Abuse. Methods 39 (3), 262-274. doi:10.1016/j.ymeth.2005.12.007

Orger, M. B., and De Polavieja, G. G. (2017). Zebrafish Behavior: Opportunities and Challenges. Annu. Rev. Neurosci. 40, 125-147. doi:10.1146/annurev-neuro071714-033857

Parichy, D. M., Elizondo, M. R., Mills, M. G., Gordon, T. N., and Engeszer, R. E. (2009). Normal Table of Postembryonic Zebrafish Development: Staging by Externally Visible Anatomy of the Living Fish. Dev. Dyn. 238 (12), 2975-3015. doi:10.1002/dvdy.22113

Petersen, B. D., Pereira, T. C. B., Altenhofen, S., Nabinger, D. D., Ferreira, P. M. D. A., Bogo, M. R., et al. (2021). Antibiotic Drugs Alter Zebrafish Behavior. Comp. Biochem. Physiol. Part. - C Toxicol. Pharmacol. 242, 108936. doi:10.1016/ j.cbpc. 2020.108936

Polverino, G., Bierbach, D., Killen, S. S., Uusi-Heikkilä, S., and Arlinghaus, R. (2016). Body Length Rather Than Routine Metabolic Rate and Body Condition Correlates with Activity and Risk-Taking in Juvenile Zebrafish Danio Rerio. J. Fish. Biol. 89 (5), 2251-2267. doi:10.1111/jfb.13100

Ricci, L., Summers, C. H., Larson, E. T., O’Malley, D., and Melloni, R. H. (2013). Development of Aggressive Phenotypes in Zebrafish: Interactions of Age, Experience and Social Status. Anim. Behav. 86 (2), 245-252. doi:10.1016/ j.anbehav.2013.04.011

Roberts, A. C., Bill, B. R., and Glanzman, D. L. (2013). Learning and Memory in Zebrafish Larvae. Front. Neural Circuits 7 (JUL), 126-211. doi:10.3389/ fncir.2013.00126

Sager, J. J., Bai, Q., and Burton, E. A. (2010). Transgenic Zebrafish Models of Neurodegenerative Diseases. Brain Struct. Funct. 214, 285-302. doi:10.1007/ s00429-009-0237-1

Sallinen, V., Sundvik, M., Reenilä, I., Peitsaro, N., Khrustalyov, D., Anichtchik, O., et al. (2009). Hyperserotonergic Phenotype after Monoamine Oxidase Inhibition in Larval Zebrafish. J. Neurochem. 109 (2), 403-415. doi:10.1111/ j.1471-4159.2009.05986.x

Schnörr, S. J., Steenbergen, P. J., Richardson, M. K., and Champagne, D. L. (2012). Measuring Thigmotaxis in Larval Zebrafish. Behav. Brain Res. 228 (2), 367-374. doi:10.1016/j.bbr.2011.12.016

Scott, G. R., and Sloman, K. A. (2004). The Effects of Environmental Pollutants on Complex Fish Behaviour: Integrating Behavioural and Physiological Indicators of Toxicity. Aquat. Toxicol. 68 (4), 369-392. doi:10.1016/ j.aquatox.2004.03.016

Senger, M. R., Rico, E. P., Dias, R. D., Bogo, M. R., and Bonan, C. D. (2004). Ecto-5'Nucleotidase Activity in Brain Membranes of Zebrafish (Danio Rerio). Comp. Biochem. Physiol. B Biochem. Mol. Biol. 139 (2), 203-207. doi:10.1016/ j.cbpc.2004.07.011

Serra, E. L., Medalha, C. C., and Mattioli, R. (1999). Natural Preference of Zebrafish (Danio Rerio) for a Dark Environment. Braz. J. Med. Biol. Res. 32 (12), 1551-1553. doi:10.1590/s0100-879x1999001200016

Shao, E., Bai, Q., Zhou, Y., and Burton, E. A. (2017). Quantitative Responses of Adult Zebrafish to Changes in Ambient Illumination. Zebrafish 14 (6), 508-516. doi:10.1089/zeb.2017.1468

Siebel, A. M., Menezes, F. P., Da Costa Schaefer, I., Petersen, B. D., and Bonan, C. D. (2015). Rapamycin Suppresses PTZ-Induced Seizures at Different Developmental Stages of Zebrafish. Pharmacol. Biochem. Behav. 139 Pt B, 163-168. doi:10.1016/j.pbb.2015.05.022

Sison, M., and Gerlai, R. (2011). Behavioral Performance Altering Effects of MK801 in Zebrafish (Danio Rerio). Behav. Brain Res. 220 (2), 331-337. doi:10.1016/ j.bbr.2011.02.019

Spence, R. (2011). "Zebrafish Ecology and Behaviour," in Zebrafish Models in Neurobehavioral Research. Editors A. V. Kalueff and J. M. Cachat (Totowa, NJ: Humana Press), 1-46. doi:10.1007/978-1-60761-922-2_1

Stednitz, S. J., and Washbourne, P. (2020). Rapid Progressive Social Development of Zebrafish. Zebrafish 17 (1), 11-17. doi:10.1089/zeb.2019.1815

Steenbergen, P. J., Richardson, M. K., and Champagne, D. L. (2011). Patterns of Avoidance Behaviours in the Light/Dark Preference Test in Young Juvenile Zebrafish: A Pharmacological Study. Behav. Brain Res. 222 (1), 15-25. doi:10.1016/j.bbr.2011.03.025

Stewart, A. M., Braubach, O., Spitsbergen, J., Gerlai, R., and Kalueff, A. V. (2014). Zebrafish Models for Translational Neuroscience Research: From Tank to Bedside. Trends Neurosci. 37 (5), 264-278. doi:10.1016/j.tins.2014.02.011 
Stewart, A., Maximino, C., Marques De Brito, T., Herculano, A. M., Gouveia, A., Jr, Morato, S., et al. (2011). "Neurophenotyping of Adult Zebrafish Using the Light/Dark Box Paradigm," in Zebrafish Neurobehavioral Protocols. Editors A. V. Kalueff and J. M. Cachat (Totowa, NJ, USA: Humana Press), 157-167. doi:10.1007/978-1-60761-953-6_13

Tal, T., Yaghoobi, B., and Lein, P. J. (2020). Translational Toxicology in Zebrafish. Curr. Opin. Toxicol. 23-24, 56-66. doi:10.1016/j.cotox.2020.05.004

Valente, A., Huang, K. H., Portugues, R., and Engert, F. (2012). Ontogeny of Classical and Operant Learning Behaviors in Zebrafish. Learn. Mem. 19 (4), 170-177. doi:10.1101/lm.025668.112

Westerfield, M. (2000). Zebrafish Book: A Guide for the Laboratory Use of Zebrafish (Danio R). 4th ed. Eugene: University of Oregon Press.

Wullimann, M. F., and Mueller, T. (2004). Teleostean and Mammalian Forebrains Contrasted: Evidence from Genes to Behavior. J. Comp. Neurol. 475 (2), 143-162. doi:10.1002/cne.20183

Yashina, K., Tejero-Cantero, Á., Herz, A., and Baier, H. (2019). Zebrafish Exploit Visual Cues and Geometric Relationships to Form a Spatial Memory. iScience 19, 119-134. doi:10.1016/j.isci.2019.07.013
Conflict of Interest: The authors declare that the research was conducted in the absence of any commercial or financial relationships that could be construed as a potential conflict of interest.

Publisher's Note: All claims expressed in this article are solely those of the authors and do not necessarily represent those of their affiliated organizations, or those of the publisher, the editors and the reviewers. Any product that may be evaluated in this article, or claim that may be made by its manufacturer, is not guaranteed or endorsed by the publisher.

Copyright (C) 2022 Petersen, Bertoncello and Bonan. This is an open-access article distributed under the terms of the Creative Commons Attribution License (CC BY). The use, distribution or reproduction in other forums is permitted, provided the original author(s) and the copyright owner(s) are credited and that the original publication in this journal is cited, in accordance with accepted academic practice. No use, distribution or reproduction is permitted which does not comply with these terms. 